\title{
Canadian best practice recommendations for the management of amyotrophic lateral sclerosis
}

\author{
Christen Shoesmith MD, Agessandro Abrahao MD MSc, Tim Benstead MD, Marvin Chum MD MSc, \\ Nicolas Dupre MD MSc, Aaron Izenberg MD, Wendy Johnston MD, Sanjay Kalra MD, Desmond Leddin MB MSc, \\ Colleen O'Connell MD, Kerri Schellenberg MD MMedEd, Anu Tandon MD, Lorne Zinman MD MSc
}

Cite as: CMAJ 2020 November 16;192:E1453-68. doi: 10.1503/cmaj.191721

This guideline is available in French at www.cmaj.ca/lookup/doi/10.1503/cmaj.191721-f

CMAJ Podcasts: author interview (in English) at www.cmaj.ca/lookup/doi/10.1503/cmaj.191721/tab-related-content

A myotrophic lateral sclerosis (ALS) is a debilitating, progressive disease with degeneration of motor neurons in the brain and spinal cord causing weakness, muscle atrophy, fasciculations and spasticity. ${ }^{1}$ Onset in the limbs, with extremity weakness and impairment in mobility, is the most common presentation, occurring in about $70 \%$ of patients. ${ }^{2}$ Bulbar onset with oropharyngeal muscle involvement affecting swallowing and speech occurs in about $25 \%$ of cases. ${ }^{2}$ In addition to motor impairment, degeneration in the frontal and temporal lobes, resulting in cognitive or behavioural impairments, occurs in up to $50 \%$ of patients. ${ }^{3}$ Over time, strength progressively declines, and patients typically die from respiratory failure within 5 years of diagnosis. ${ }^{2}$ Despite increased research efforts in recent years, treatment options for ALS remain limited, and patient care is focused primarily on managing symptoms and optimizing function and quality of life. ${ }^{2}$

An estimated 3000 Canadians are currently living with ALS. ${ }^{4,5}$ Advocacy groups and clinicians caring for patients with ALS have strongly supported the development of best practice recommendations for the care and management of these patients in Canada. Although ALS clinical practice guidelines have been published in the United States ${ }^{6,7}$ and in Europe, ${ }^{8,9}$ to date there have been no published guidelines explicitly for the care of patients with ALS in Canada.

In addition to providing an update on the evolving standard of care in ALS, the best practice recommendations in this guideline serve to address several issues important to Canadians, such as caregiver support, medication alignment and medical assistance in dying (MAiD). Developing the first Canadian ALS guideline is a critical step in an iterative process whereby these recommendations can be updated as evidence evolves, and research priorities can be identified and prioritized to fill knowledge gaps.

Because the rigorous standards of evidence-based medical recommendations are not met in most areas of ALS care, many

\section{KEY POINTS}

- Management of patients living with amyotrophic lateral sclerosis (ALS) requires specialized multidisciplinary holistic care.

- Disease-modifying pharmacologic therapies to treat ALS include riluzole and edaravone.

- Close attention to nutritional support and respiratory care is required for optimal care in ALS.

- Multiple treatments are available to ease the symptoms of ALS.

- Palliative care and caregiver support are important components of assisting patients along their journey with ALS.

of the recommendations presented are expert consensus on good practice. Typically, symptom management in ALS is extrapolated from evidence in other disease states. The recommendations presented in this guideline are based on best available evidence and expert consensus on best practices, and thus reflect the real-life experiences of Canadian clinicians caring for patients with ALS. This article is a summary of the full guideline, which is available on the ALS Canada website (www.als.ca/ bpr-appendix).

\section{Scope}

The purpose of this guideline is to provide ALS clinicians, allied health professionals and primary care providers with best practice recommendations for the care and management of patients living with ALS in Canada, inclusive of all genders, ages and stages of the disease. This guideline is intended to develop a national standard to improve quality of care for patients, families and caregivers living with ALS. Advocacy groups (e.g., ALS Society 
of Canada [ALS Canada], provincial ALS societies), health authorities, governments and policy-makers will be better able to establish benchmarks and advocate for standards of care.

\section{Recommendations}

The care and management of patients with ALS should always be patient focused, with attention to holistic and emotional aspects of well-being. It is the patient who ultimately decides on their treatment; this includes the option of declining interventions.

The recommendations for the management of patients with ALS in Canada are in Table 1, grouped by topic, and indicating the level of evidence. If evidence was insufficient or absent for a key question, we made recommendations based on expert consensus through review of the available literature and clinical experience in ALS or extrapolated from treatment of other more common diseases.

Table 1 (part 1 of 6): Recommendations with level of evidence grade for the management of patients with amyotrophic lateral sclerosis*

\section{Recommendations}

\section{Communication of diagnosis}

- The approach to communicating the diagnosis should be tailored to the patient's individual needs (EC).

- The diagnosis of ALS should be confirmed by a neurologist or physiatrist with training and expertise in ALS (EC). Patients referred for confirmation of an initial diagnosis of ALS should be seen in an ALS specialty clinic within 4 weeks (EC). Timely clinical contact by the specialty ALS clinic after confirmation of diagnosis is recommended (EC).

- Discussion about ALS treatments and ALS research should occur. Patients should be provided with written information about ALS resources (paper- or Internet-based) and encouraged to register with their local and national ALS society (EC).

- Discussions on prognosis are important and should be tailored to the individual but need not be discussed at initial diagnosis unless specifically requested $(\mathrm{EC})$.

\section{Disease-modifying therapies}

- Disease-modifying therapies should be prescribed by clinicians with experience in managing patients with ALS (EC).

- Riluzole:

- Riluzole has demonstrated efficacy in improving survival in ALS (level A).

- There is evidence that riluzole prolongs survival by a median duration of 3 months (level A).

- Riluzole should be started soon after the diagnosis of ALS (EC).

- Regular monitoring of potential adverse effects of riluzole is important (EC).

- There is insufficient evidence to suggest that riluzole loses clinical efficacy with progression of disease, including development of respiratory insufficiency (EC).

- Edaravone:

- In a select group of patients, intravenous edaravone has been shown to slow decline on the ALSFRS-R scores compared against intravenous placebo, over a 6-month period (level B). (These patients have shown benefit from edavarone: disease duration < 2 y, FVC $>80 \%$, all ALSFRS-R subcomponents scores > 2, and demonstrated steady decline in the ALSFRS-R over a 3-mo interval.)

- Evidence for benefit of intravenous edaravone at other stages of ALS has not been demonstrated (EC).

- As with any other therapies, individualized goals, risks and benefits should be carefully considered and discussed before intravenous edaravone is initiated (EC).

- Physicians are encouraged to have an open dialogue with their patients about the potential risks and benefits of unapproved therapies (EC).

\section{Multidisciplinary care}

- Patients with ALS should be referred to specialized ALS multidisciplinary clinics for optimized health care delivery (level B).

- Patients and health care authorities should be educated on the rationale for patient attendance at a multidisciplinary clinic. Benefits include:

- Survival benefit (level B).

- Fewer and shorter hospital admissions than patients not attending such clinics (level B).

- Increased use of adaptive equipment (level C).

- Increased use of riluzole, percutaneous feeding tubes and NIV (level B).

- Enhanced QOL (level C).

- Multidisciplinary care should be delivered through a team-based approach, with physicians and other health professionals addressing issues including communication, nutrition, swallowing, mobility, activities of daily living, respiratory care, cognition, psychosocial issues, medical management and end-of-life care (EC).

- The frequency of multidisciplinary clinic visits will be dictated by the patient's needs and rate of progression (EC).

- A dedicated nurse or other clinic allied health care professional should be available to support patients and their family members for ALS issues between clinic visits (EC).

- Telemedicine and telehealth monitoring are feasible and may be able to supplement clinic-based multidisciplinary care (level C). 
Table 1 (part 2 of 6): Recommendations with level of evidence grade for the management of patients with amyotrophic lateral sclerosis*

\section{Recommendations}

\section{Respiratory management}

Screening

- Patients with ALS need regular respiratory monitoring at baseline and every 3 months, or as clinically indicated (EC). Regular respiratory monitoring should include:

- Symptom review, including dyspnea, orthopnea and morning headaches (level C).

- Measurement of sitting FVC or slow vital capacity (level B).

- One or more of the following: SNIP, supine FVC or MIP (level C).

- Arterial blood gases, venous blood gas or transcutaneous $\mathrm{CO}_{2}$, when hypercapnia is suspected or when bulbar impairment precludes accurate testing (level C).

- PCF measurement to assess cough effectiveness (level C).

- Nocturnal oximetry or overnight polysomnography, when symptomatic sleep-disordered breathing is suspected and other daytime indications for NIV initiation are not present (level C).

Ventilation

- NIV is the standard of care to treat respiratory insufficiency in ALS, both to lengthen survival and treat symptoms (level B).

- Criteria for NIV initiation are any of the following:

- Symptoms of respiratory insufficiency, including orthopnea (level B).

- $\mathrm{SNIP} \leq 40 \mathrm{~cm} \mathrm{H}_{2} \mathrm{O}$ or MIP $\leq 40 \mathrm{~cm} \mathrm{H}_{2} \mathrm{O}$ (level C).

- Upright reliable† FVC $<65 \%$ (EC).

- FVC sitting or supine $<80 \%$ with symptoms or signs of respiratory insufficiency (level B).

- Daytime hypercapnia $\mathrm{pCO}_{2}>45 \mathrm{~mm} \mathrm{Hg}$ (level B).

- Abnormal nocturnal oximetry or symptomatic sleep-disordered breathing (level B).

- A respiratory specialist should be consulted to initiate NIV (EC).

- In any patient with the above indications, NIV should be initiated within 4 weeks. Severely symptomatic patients will need more urgent initiation. An overnight polysomnogram is not required for initiation of NIV (EC).

- Ensure in-homeł NIV respiratory support for education, titration and troubleshooting (EC).

- Patients should be informed that use of NIV may change the survival trajectory in ALS and the end-of-life experience (EC).

- NIV enhances QOL in patients with ALS who have respiratory insufficiency (level B).

- There should be ongoing assessments by a specialized respiratory therapist who can optimize modes, pressure and interfaces of NIV. Monitoring should include device download and may include nocturnal oximetry (level C).

- Oxygen should not be considered a routine treatment for chronic respiratory insufficiency. In patients with ALS with acute hypoxemia, management of respiratory insufficiency with NIV needs to be considered first. If hypoxemia remains after optimal NIV pressure is applied, the etiology of the hypoxia needs to be assessed and supplemental oxygen can be considered (EC).

- Diaphragm pacing should not be used in ALS because it is not effective and likely harmful in patients with ALS (level B).

- NIV is the recommended treatment for ventilation even when ventilation is required 24 hours per day (EC).

- Mouthpiece ventilation can be considered in carefully selected patients as a form of NIV during the day in addition to nocturnal NIV (EC).

- In respiratory impairment that cannot be effectively managed by NIV, invasive ventilation is an option in carefully selected patients. Discussions pertaining to goals of care and advanced directives should occur well in advance of respiratory failure (EC).

- Patients need to understand that ALS will continue to progress even with ventilatory support (EC).

- Advanced care planning discussions should include explicit information about all respiratory interventions. Discussions should include the fact that intubation may be irreversible depending on the disease stage, and palliative options for breathlessness (please refer to the Palliative Care section). Discussions should also include the option of removing any treatment that has been initiated (EC).

- Tracheostomy can be considered for upper airway obstruction with vocal cord paresis; however, discussions of long-term invasive ventilation should also occur (EC).

Airway clearance management

- Lung volume recruitment strategies (level C) and manual assisted coughing (EC) should be initiated when patients report difficulty clearing airway secretions.

- MIE twice daily should be considered for secretion clearance in patients with ALS who have reduced PCF (<270 L/min). Increased MIE frequency should occur during an acute chest infection (EC).

- Ensure in-homeł respiratory support of MIE for education, titration and troubleshooting (EC).

- Pharmacotherapy with mucolytics (i.e., guaifenesin or N-acetylcysteine), a $\beta$-receptor antagonist (e.g., metoprolol or propranolol), nebulized saline or nebulized ipratropium can be considered (EC). 
Table 1 (part 3 of 6): Recommendations with level of evidence grade for the management of patients with amyotrophic lateral sclerosis*

\section{Recommendations}

\section{Nutritional management}

Monitoring and enteral intervention

- Nutritional status should be monitored by weight and BMI every 3 months, or as clinically indicated (level B); TDEE may be considered (level B).

- Nutritional interventions, including dietary alteration and consideration of referral for enteral tube insertion, are indicated at diagnosis or at follow-up if there is: (1) increased risk of aspiration despite consistency modifications and compensatory recommendations (EC); (2) $\geq 5 \%-10 \%$ reduction in weight from usual or baseline weight (level C); (3) $\geq 1$-point reduction in BMI from usual or baseline BMI (level B); (4) BMI < 18.5 (level B); or (5) TDEE exceeds daily energy intake (EC).

- Information regarding potential benefits and risks of enteral feeding tubes should be provided early in the course of ALS management (EC).

- A decrease in FVC approaching 50\% should prompt consideration of referral for enteral tube insertion, even in the absence of dysphagia. An FVC $<50 \%$ should not necessarily preclude the recommendation of enteral feeding tube insertion as long as respiratory status is carefully monitored during and after the procedure (level C). NIV may improve safety of RIG or PEG insertion in patients with respiratory impairment (EC).

- Regular monitoring of swallowing safety should be performed by a certified swallowing clinician (level B). Objective measures of swallowing impairment (modified barium swallow or FEES) can be used early and during the course of ALS management (EC).

Maximum allowable delay for PEG or RIG

- Once a decision is made to insert an enteral feeding tube, insertion should be performed within 4 weeks. The ALS team should have access to endoscopists or radiologists who have interest and expertise in tube insertion (EC).

Feeding tube insertion

- There is insufficient evidence to recommend PEG or RIG as the usual procedure for gastrostomy insertion (level C). There is weak evidence that RIG may be safer in patients with ventilatory impairment, as RIG does not require substantial sedation (EC).

- Once a feeding tube is placed, an experienced clinician (endoscopist or radiologist) should be readily available to address immediate and late tube complications. There should be regular support by a registered dietitian with respect to the enteral feeds prescribed (EC).

- Nasogastric tube feeding is not a preferred long-term option and should be reserved for those patients where no other procedure is possible and enteral nutrition is still desired (level C).

Diet modifications and nutrition support

- High-calorie diets can be used to improve nutritional indicators (level B) and possibly survival (level C). High-calorie and high-carbohydrate diets may be better than high-calorie and high-fat diets (level B).

- Parenteral nutrition is a potential source of nutrition in patients who cannot successfully have an enteral nutrition source; its use should be reserved for exceptional circumstances (EC).

\section{Venous thromboembolism}

- There is likely an increased risk of VTE in patients with ALS. The risk appears heightened in ALS with leg onset and in patients with poor mobility (EC).

- Clinicians are encouraged to consider VTE as a potential cause for new leg pain or new leg swelling in patients with ALS (EC).

- There is no evidence to suggest screening for thromboembolism in asymptomatic patients with ALS (EC).

- VTE prophylaxis has not been evaluated in patients in ALS and is not recommended in patients who have not been admitted to hospital (EC).

- If VTE occurs in a patient with ALS, they should be anticoagulated as per standard VTE guidelines (EC).

\section{Medication alignment}

- Primary care physicians and specialists should perform intermittent medication reviews and consider discontinuing any nonessential medications (EC).

- Symptom management medications should be continued (EC).

- Primary prevention medications should be discontinued if duration of effect is longer than the expected survival (EC).

- Patients and health care professionals can be reassured that premorbid statin administration does not appear to contribute to the development of ALS (level B).

- There is insufficient evidence to recommend discontinuation of statins in all patients with ALS. Discontinuation of statins may be considered based on the patient's expected survival and their cardiovascular risk (EC).

The following sections provide background and expand on selected recommendations with supporting evidence. The evidence tables in Appendix $\mathrm{C}$ of the full guideline (available at www.als.ca/bpr-appendix) provide details of supporting evidence for all recommendations listed in Table 1. For a complete discussion of the evidence supporting the recommendations, please see Appendix A (www.als.ca/bpr-appendix).

\section{Communication of diagnosis}

The manner in which the diagnosis of ALS is delivered is a source of discontent for many patients and caregivers. ${ }^{10,11}$ Recommendations have been formulated that outline a comprehensive approach to diagnosis delivery in the context of ALS. ${ }^{8}$ One of the most important concepts for clinicians to consider is tailoring the diagnosis delivery to the individual needs of the patient. If a 
Table 1 (part 4 of 6): Recommendations with level of evidence grade for the management of patients with amyotrophic lateral sclerosis*

\section{Recommendations}

\section{Symptom management}

\section{Pain}

- Pain is a recognized consequence of ALS, with many potential causes (EC).

- Patients must be queried regularly about pain symptoms. Pain should be regularly assessed and treatments should be tailored toward the specific cause (EC).

Fasciculations

- In most patients, fasciculations do not need medication management (EC).

- If fasciculations cause substantial distress, gabapentin can be considered (level C).

Sialorrhea

- Anticholinergic medications are the first-line therapy of sialorrhea. Individual medication choices should be tailored to patient factors (EC).

- If one anticholinergic medication is ineffective, switching to another anticholinergic medication should be considered (EC).

- Oral suction can be used as an adjunct therapy in managing sialorrhea (EC).

- Botulinum toxin is effective for management of sialorrhea in ALS (level A). It can be used as second-line therapy and should be considered after feeding tube insertion because of the theoretical risk of worsening swallowing or airway integrity (EC).

- Focal salivary gland radiation is an option for management of sialorrhea (level C) as second- or third-line therapy.

Pseudobulbar affect

- Patients and families should be educated that pseudobulbar affect is a symptom of ALS and does not necessarily represent a symptom of depression or impaired cognition (EC).

- Pseudobulbar affect does not require treatment unless it is distressing to the patient (EC).

- If treatment is warranted, medications that may co-treat concomitant symptoms (e.g., amitriptyline for sleep and mood effect, SSRI for depression) may be considered (EC).

- Dextromethorphan $(20 \mathrm{mg})$ combined with quinidine $(10 \mathrm{mg})$ can be used for treatment of pseudobulbar affect (level B).

Spasticity

- Stretching can be useful for managing spasticity (level C).

- If pharmacologic management of spasticity is required, baclofen, tizanidine, botulinum toxin, benzodiazepines and cannabinoids could be considered (EC).

- There is insufficient evidence to recommend intrathecal baclofen for spasticity management in patients with ALS (EC).

cramps

- Muscle cramps need to be differentiated from other causes of pain (EC).

- First-line management could include tonic water, gabapentin and baclofen (EC).

- Second-line treatment could include quinine, levetiracetam and mexiletine (EC).

Depression

- Depression should be treated in ALS, as it has a substantial impact on patient well-being (EC).

- SSRIs or SNRIs can be used to treat depression in ALS (EC).

- Nonpharmacologic supports could be considered, such as those offered through psychology, social work, psychiatry or spiritual care (EC).

Anxiety

- Anxiety should be treated in ALS as it has a substantial impact on patient well-being (EC).

- It is important to determine if anxiety is related to respiratory insufficiency and, if present, treat appropriately (EC).

- If depression is concurrently present, an SSRI should be prescribed (EC).

- Benzodiazepines can exacerbate respiratory insufficiency (EC).

- Nonpharmacologic supports can be considered, such as those offered through psychology, social work, psychiatry or spiritual care (EC).

Insomnia

- There are multiple causes of insomnia, such as respiratory insufficiency and depression, that should be appropriately investigated (EC).

- Respiratory investigations and sleep studies could be considered to determine the type and cause of insomnia (EC).

- Pharmacologic management of insomnia will depend on the cause (EC).

Fatigue

- Reversible causes of fatigue should be considered, such as respiratory insufficiency, sleep disorders, depression, medication adverse effects and riluzole use (EC).

- In patients developing fatigue while taking riluzole, reducing or discontinuing the drug may be considered (level C).

- Having an occupational therapist discuss energy conservation techniques with patients may be considered (EC). 
Table 1 (part 5 of 6): Recommendations with level of evidence grade for the management of patients with amyotrophic lateral sclerosis*

\section{Recommendations}

\section{Dysarthria}

- Patients with dysarthria should be regularly followed by a speech language pathologist to ensure timely communication interventions (EC).

- Use of augmentative and alternative communication devices should be offered to eligible patients in early disease stages (EC). Patients in later disease stages will also benefit from communication devices and strategies (EC).

- The choice of communication devices should be tailored to the patient's needs and abilities (EC). Patients with cognitive impairment may need individualized strategies for communication (EC).

- Augmentative and alternative communication strategies may reduce caregiver stress (EC).

- Voice amplification should be offered to patients with reduced vocal projection (EC).

- Voice banking should be offered to appropriate patients (EC).

- Providing access to different modes of communication, including social media, can allow independence, participation and better QOL (EC).

\section{Exercise}

- In early ALS, regular moderate-intensity exercise is probably beneficial for function and QOL (level B). A personalized exercise program, including strength and aerobic training, should be suggested to patients who are able to participate (EC):

- Submaximal effort for resistance should be encouraged.

- Moderate-intensity physical activities are those that will cause adults to sweat a little and to breathe harder.

- Moderate-intensity exercise is well tolerated and not harmful in ALS (level B):

- Post-exercise fatigue or pain should resolve in 30 minutes and not interfere with daily activities; the exercise program should be adjusted otherwise.

- A regular stretching and range-of-motion program is recommended for management of spasticity (level C), pain (EC) and prevention of contractures (EC).

- Stretching and range-of-motion exercise can be done independently (active), with assistance (passive) or in combination (active-assist)

\section{Cognition and behaviour}

- Screening for cognitive and behavioural impairment should be performed in patients with ALS early in their disease (level B).

- If there is concern about cognition or behaviour at any point, specific assessments should take place with the person and their family members or caregiver, as appropriate (EC).

- There are no studies on the use of pharmacologic agents to manage cognitive or behavioural impairment in ALS.

- Because the presence of frontotemporal dementia negatively affects survival, ACP should be done early in the disease (EC).

- The presence of cognitive or behavioural impairment should not necessarily preclude the recommendations for NIV or gastrostomy insertion. However, the challenges of intervention compliance with cognitive or behavioural impairment should be discussed with the patient and family before deciding to proceed with an intervention (EC).

- A multidisciplinary approach can be considered to manage particularly problematic behaviours. Involving a behavioural specialist (such as an occupational therapist or psychologist) or psychiatrist for assistance may be considered (EC).

\section{Caregivers}

- Health care providers should be attentive to the needs and emotional well-being of caregivers. Caregivers should be involved in planning for the impact of ALS on both the patient and themselves (EC).

- Multidisciplinary clinics should be aware of the financial strain on caregivers and provide information on existing relief programs where possible (EC).

- Assessment of caregiver burden, coping strategies, mood and family dynamics would assist in identifying caregivers and families in need of respite and supportive services. Local ALS societies may have resources for family members and caregivers (EC).

patient is overwhelmed by the diagnosis of ALS, then the diagnosis could be delivered in a stepwise fashion, without divulging all of the information at once. ${ }^{12}$ Conversely, patients may feel that they did not receive enough information when receiving their diagnosis. Patients and caregivers wish to be informed about current research, treatments and prognosis when receiving a diagnosis of ALS. ${ }^{13}$

In a study of satisfaction with the manner of disclosure of the diagnosis of ALS, $41 \%$ of patients indicated that they received insufficient information, and one-third stated that they were not given a contact for follow-up. ${ }^{10}$ Furthermore, about $75 \%$ of patients and caregivers had questions that arose immediately after they received the initial diagnosis. ${ }^{11}$ These findings highlight the need for clinicians to address sources of information, community support and provide timely follow-up after the diagnosis is first discussed. Patients report better satisfaction with the delivery of an ALS diagnosis if they believe that the clinician has understood their feelings. ${ }^{13}$ An additional source of frustration for patients was the delay in receiving confirmation of a diagnosis, including wait times to see an ALS specialist. ${ }^{10}$ The working 
Table 1 (part 6 of 6): Recommendations with level of evidence grade for the management of patients with amyotrophic lateral sclerosis*

\section{Recommendations}

\section{Palliative care}

Timing

- Palliative care of patients with ALS can be provided throughout the disease course by ALS clinic staff, palliative care practitioners and family physicians (EC).

- Palliative care should be introduced if there is severe physical (i.e., pain, dysphagia or dyspnea), psychosocial or existential distress (EC).

- To ensure integrated continuity of care, community palliative care services could be introduced before advanced-stage ALS (EC).

Treatments

- Clinicians must clarify with their patient who is experiencing breathlessness whether the goal of care is prolonging life versus comfort-focused care for a good death (EC).

- Clinicians should assess and relieve factors contributing to breathlessness, such as oral secretions and anxiety (EC).

- Opioids can be titrated to relieve breathlessness (EC).

- Air flow across the face to help with breathlessness may be considered (EC).

- Conversations about ACP should be initiated early in the disease or whenever the patient inquires. Ongoing discussions about ACP and goals of care should be part of routine ALS follow-up (EC).

- Patients should be encouraged to discuss their preferences about end-of-life care with family members and caregivers (EC).

- Palliative care should be integrated into routine patient management before the terminal phase of ALS (EC).

- Use of NIV and PEG tubes should be continued in palliative care for symptom relief and QOL, as dictated by patient preference (EC).

Withdrawal of ventilatory support

- Withdrawal of continuous ventilatory support should be performed only after consultation and planning with a health care professional with expertise in ventilation withdrawal and palliative sedation (EC).

- Adequate anticipatory symptom control with opioids and benzodiazepines should be achieved before withdrawal of ventilation occurs (EC).

- Debriefing and psychosocial support for family and health care providers should be offered (EC).

Bereavement

- Psychosocial support for bereaved caregivers should be provided. Early discussion about and support for the bereavement process could be initiated even before the patient's death (EC).

Medical assistance in dying

- Discussions about MAiD should be directed to a physician or nurse practitioner, abiding by regional guidelines (EC).

- Physicians caring for patients with ALS are required to provide access to information about MAiD when requested (EC).

- Clinicians should not assume that questions about MAiD constitute a request for MAiD. However, questions about MAiD should also open a discussion about end-of-life care and ACP (EC).

- Patients pursuing MAiD should be provided concurrent palliative and supportive care (EC).

Organ donation

- Patients with ALS may be accepted as solid organ donors, as determined by their local organ donation organization (EC).

- Patients with ALS cannot donate tissue, such as corneas, skin or bone (EC).

- Clinics should direct inquiries about donation to their provincial organ donation organization (EC).

- Patients may be able to donate their tissues upon death for ALS research (EC).

Note: $\mathrm{ACP}=$ advanced care planning, ALS = amyotrophic lateral sclerosis, ALSFRS-R = Amyotrophic Lateral Sclerosis Functional Rating Scale-Revised, $\mathrm{BMI}=$ body mass index, EC = expert consensus, $\mathrm{FEES}=$ fibreoptic endoscopic evaluation of swallowing, $\mathrm{FVC}=$ forced vital capacity, $\mathrm{MAiD}=$ medical assistance in dying, $\mathrm{MIE}=$ mechanical insufflation-exsufflation, $\mathrm{MIP}=$ maximal inspiratory pressure, $\mathrm{NIV}=$ noninvasive ventilation, $\mathrm{PCF}=$ peak cough flow, $\mathrm{pCO}_{2}=$ partial pressure of carbon dioxide, $\mathrm{PEG}=$ percutaneous endoscopy gastrostomy,

$\mathrm{QOL}=$ quality of life, RIG = radiologically inserted gastrostomy, SNIP = sniff nasal inspiratory pressure, SNRI = serotonin-norepinephrine reuptake inhibitors, SSRI = selective serotonin reuptake inhibitor, TDEE = total daily energy expenditure, VTE = venous thromboembolism.

*See Box 2 for criteria for levels of evidence supporting the recommendations.

†If pulmonary testing is not reliable (i.e., in patients with bulbar impairment or with severe cognitive impairment), clinicians must rely on symptoms or other measures for respiratory screening

$\ddagger$ The patient’s home can be a house, apartment, long-term care facility or hospice.

group agreed that a maximum wait time of 4 weeks for a consultation to confirm a diagnosis of ALS was reasonable.

\section{Disease-modifying therapies}

Health Canada approved riluzole as a treatment for ALS in 2000. Based on a class I meta-analysis of 4 randomized controlled trials (RCTs), riluzole has a modest benefit on survival compared with placebo, with a hazard ratio (HR) of 0.84 (95\% confidence interval [Cl] 0.698 to 0.997 ), representing a $9 \%$ gain in annual probability of survival. This translates to an increase in median survival from 11.8 to 14.8 months. ${ }^{14}$ Recent registry-based cohort studies (all class III) have estimated an improvement in median survival with riluzole treatment of 7.3 months, ${ }^{15} 10$ months $^{16}$ or 12 months, ${ }^{17}$ but other studies have found no effect on survival. ${ }^{18-21}$ Findings 
from other class III cohort studies reported HR estimates of $0.34,{ }^{22}$ $0.71,{ }^{23} 0.79^{24}$ and $0.81,{ }^{25}$ which translates to an estimated absolute increase in annual survival that ranges from $10 \%$ to $50 \%$.

No controlled trials have examined whether riluzole extends life at a specific stage or all stages of ALS. A post-hoc analysis of the original dose-ranging study suggests that riluzole may be effective at prolonging survival only at later disease stages (defined by nutritional or respiratory failure sufficient to require intervention), ${ }^{26}$ but results from other cohort studies differ, showing that it may be effective only at earlier stages, ${ }^{27}$ or that its effect on survival is short lived. ${ }^{28}$ Nevertheless, decades of experience worldwide have shown riluzole to be generally well tolerated with prolonged use and with regular monitoring of liver enzymes and blood counts, as well as screening for nausea and fatigue (class I). ${ }^{14}$

Health Canada approved edaravone to treat ALS in October 2018. A single class I study in a generalized ALS population did not demonstrate overall benefit of edaravone in slowing progression of the ALS Functional Rating Scale-Revised (ALSFRS-R) score over 6 months ${ }^{29}$ but did suggest benefit in a subgroup of patients (see Table 1 for characteristics of this subgroup). This beneficial effect on the slowing of the progression of the ALSFRS-R score was subsequently confirmed in a second class I study that restricted recruitment to patients with characteristics of the subgroup from the first study. ${ }^{30}$ The second study demonstrated a mean reduction in the change in ALSFRS-R score over 6 months of 2.49 ( $95 \% \mathrm{Cl} 0.99$ to 3.98$)$. At this time, the available evidence suggests a level B evidence rating of "probably effective" in a select group of patients with ALS.

\section{Multidisciplinary care}

Patients with ALS should be regularly followed by a multidisciplinary ALS clinic, along with their primary care provider. Multidisciplinary care should be delivered through a team-based approach, with physicians and other health professionals addressing a broad range of issues, including communication, nutrition, swallowing, mobility, activities of daily living, respiratory care, cognition, psychosocial issues, medical management and end-of-life care. Patients followed through a multidisciplinary clinic have been shown to have better outcomes, including improved survival, fewer hospital admissions, increased use of adaptive equipment and enhanced quality of life, than those not followed in a multidisciplinary clinic. ${ }^{31-33}$ One prospective cohort study showed that patients followed in a multidisciplinary clinic lived 7.5 months longer than those followed in a general neurology clinic. $^{32}$

Telemedicine and telehealth monitoring are feasible and may be able to supplement clinic-based multidisciplinary care. ${ }^{34}$ Management of patients with ALS should be a collaboration between the family physician and the ALS clinic, with the ALS clinic staff available for remote consultation between patient visits.

\section{Respiratory management}

The Canadian Thoracic Society (CTS) guideline group recently reviewed the respiratory management of patients with ALS on home mechanical ventilation. ${ }^{35}$ We decided to make our recommendations on respiratory management (Table 1, Figure 1) consistent with the CTS guideline. We made a few additions to the CTS recommendations, including a statement on avoiding the use of oxygen for respiratory symptoms in patients with ALS, timing of initiation of interventions and managing secretions. We also thought it was important to adjust the minimum forced vital capacity (FVC) criterion for initiation of noninvasive ventilation in asymptomatic patients to $65 \%$ of predicted, from the CTS recommendation of $50 \%$, because available evidence suggests early initiation improves survival. ${ }^{36}$ Patients with an FVC of greater than $65 \%$ predicted can be started on noninvasive ventilation if any of the other initiation criteria are met, as consistent with the CTS guideline. Our group also unanimously agreed that if criteria for initiation of noninvasive ventilation are met, patients should be initiated on noninvasive ventilation within 4 weeks.

It is important to acknowledge that noninvasive ventilation can change the natural disease trajectory of ALS. For example, increasing reliance on noninvasive ventilation converts it into lifesupport technology. In patients reliant on noninvasive ventilation, natural death may not occur while using the technology; death may occur only if there is an active decision to discontinue the ventilation support. Patients should be counselled that they may need to take an active decision as to the timing of discontinuing the ventilatory support, unless they wish prolonged survival.

Difficulty with secretion management is common among persons living with ALS, and is a cause of distress, reduced quality of life and impairment of respiratory function. The CTS guideline did not explicitly address airway clearance management. To address this important issue, we reviewed available evidence and clinical experience. We recommend that lung volume recruitment techniques be introduced whenever patients present with symptoms of retained airway secretions or difficulty in clearing secretions. Such techniques can be combined with manual assisted coughing and be performed independently by patients or with assistance of care providers. If patients develop impaired peak cough flow ( $<270 \mathrm{~L} / \mathrm{min}$ ), then mechanical insufflation-exsufflation twice daily should be considered for secretion clearance, and more frequently during an acute respiratory infection.

We also attained consensus that providing adequate in-home respiratory support of noninvasive ventilation and mechanical insufflation-exsufflation for education, titration and troubleshooting is essential, regardless of whether the patient resides in their own home, long-term care facility or hospice.

\section{Nutrition management}

The nutrition recommendations (Table 1, Figure 2 ) largely follow those outlined in the AAN guideline. ${ }^{6}$ Differences from the AAN recommendations include the addition of an expert consensus statement on the 4-week maximum allowable delay for a feeding tube insertion after criteria have been met, and a statement on the availability of appropriate follow-up after insertion for immediate or late complications. The recommendations also include a statement about nutritional components, and note that highcalorie diets can be used to improve nutritional indicators and possibly survival. ${ }^{37,38} \mathrm{High}$-calorie and high-carbohydrate diets may be better than high-calorie and high-fat diets. ${ }^{39}$ 


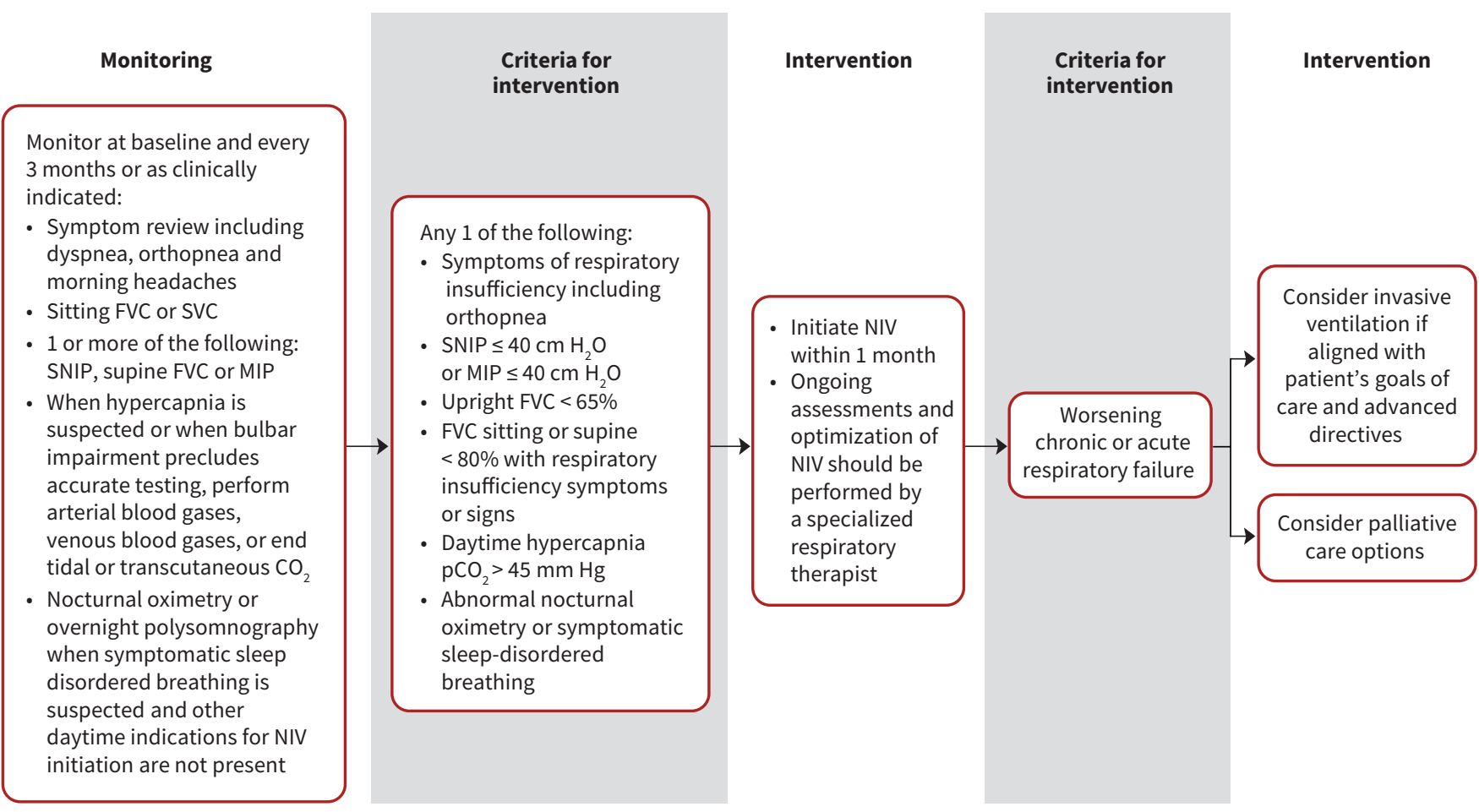

\section{B) Airway clearance}

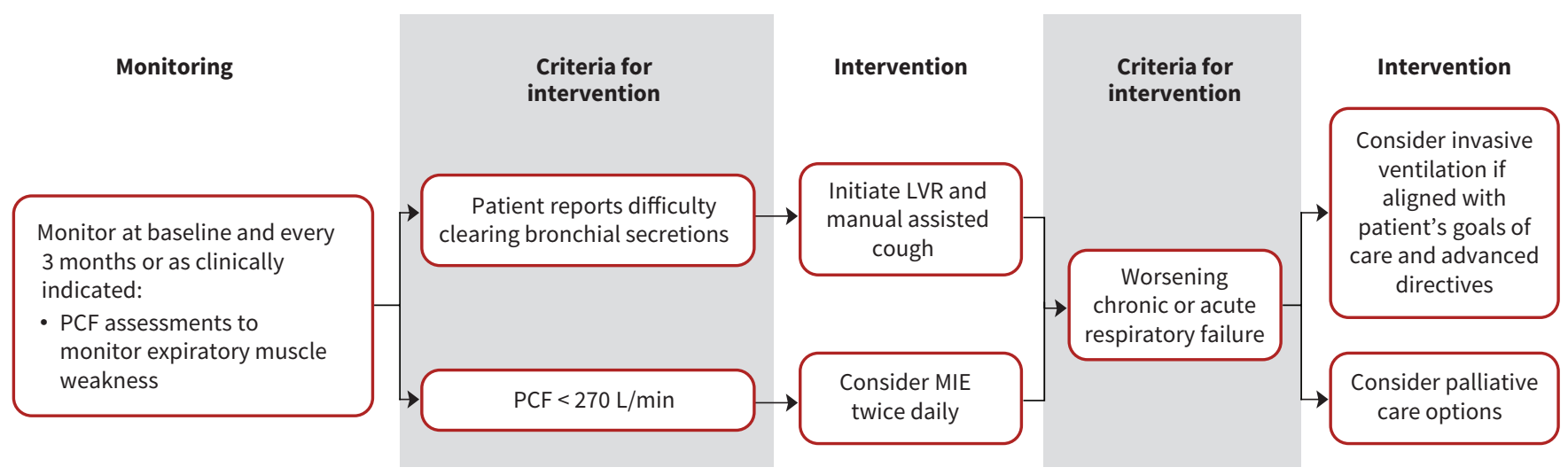

Figure 1: Respiratory decision tree: Summary of recommendations for respiratory management in patients with amyotrophic lateral sclerosis (ALS), including ventilation (A) and airway clearance (B). Note: $\mathrm{FVC}=$ forced vital capacity, $\mathrm{H}_{2} \mathrm{O}=$ water, $\mathrm{LVR}=$ lung volume recruitment, $\mathrm{MIE}=$ mechanical insufflation-exsufflation, MIP = maximal inspiratory pressure, NIV = noninvasive ventilation, $\mathrm{PCF}=$ peak cough flow, $\mathrm{pCO}_{2}=$ partial pressure of carbon dioxide, SNIP = sniff nasal inspiratory pressure, SVC = slow vital capacity.

\section{Venous thromboembolism}

There is likely an increased risk of VTE in patients with ALS.40,41 The risk appears heightened in ALS with leg onset and in patients with poor mobility. ${ }^{40}$ Despite this elevated risk, there are no studies to support primary VTE prophylaxis. At this time, primary VTE prophylaxis is not recommended because the risk-benefit ratio of potential adverse consequences from falls versus VTE prevention in patients with ALS is uncertain.

\section{Medication alignment}

When patients come to an ALS clinic, they are often on multiple medications. Some of these medications may be considered nonessential, particularly considering the average survival of patients with ALS. Through expert consensus, we developed several statements that address the need for regular review of the medications that a patient is taking and suggest discontinuation of any nonessential medications that are not providing symptomatic 


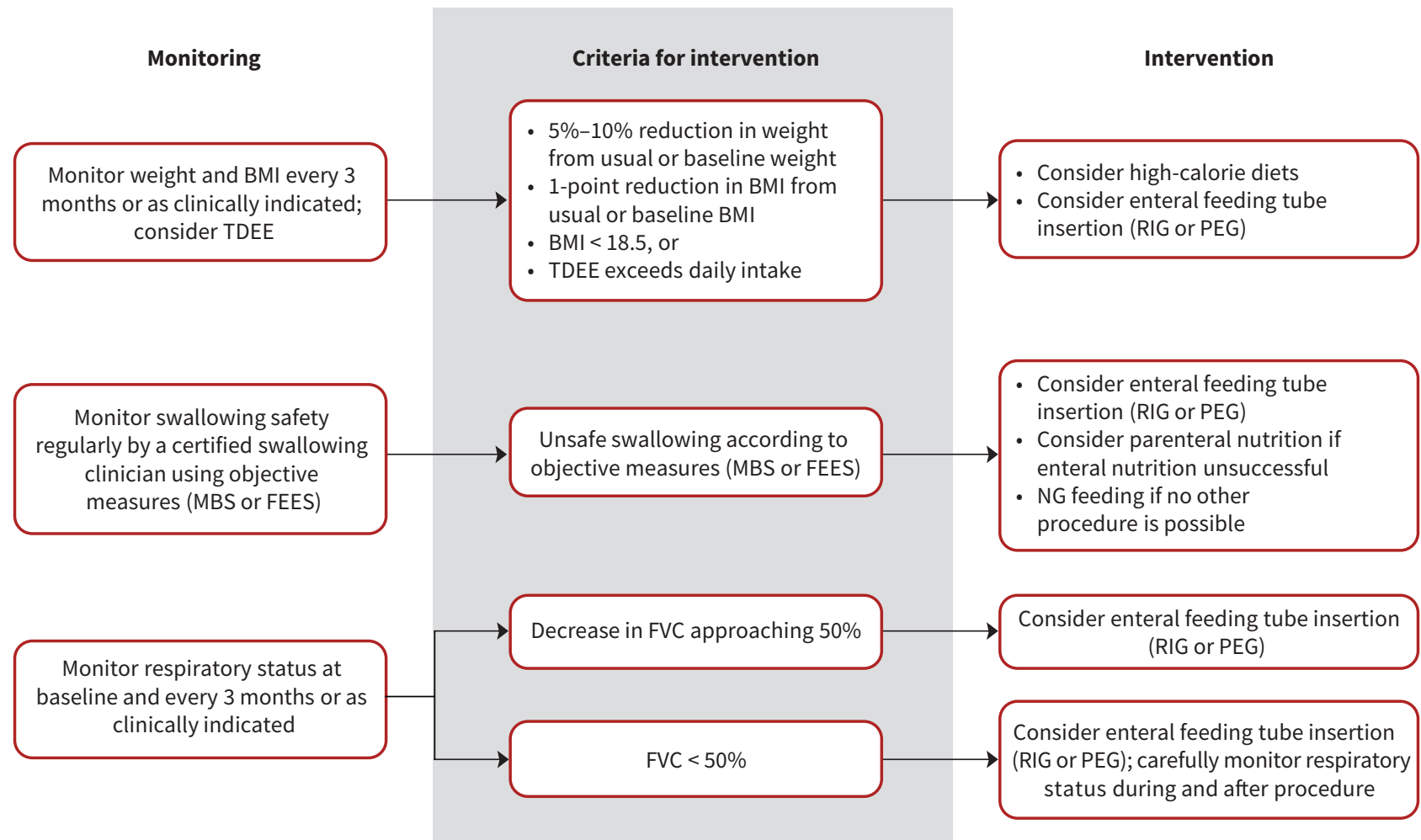

Figure 2: Nutrition decision tree: Summary of recommendations for nutritional management in patients with amyotrophic lateral sclerosis (ALS). Note: $\mathrm{BMI}=$ body mass index, FEES = fibre-optic endoscopic evaluation of swallowing, FVC = forced vital capacity, MBS = modified barium swallow, NG = nasogastric tube, $\mathrm{PEG}=$ percutaneous endoscopy gastrostomy, $\mathrm{RIG}=$ radiologically inserted gastrostomy, $\mathrm{TDEE}=$ total daily energy expenditure .

relief or appropriate therapeutic benefit in the context of an individual patient's expected survival.

\section{Symptom management}

Patients with ALS often have multiple uncomfortable symptoms that severely impair quality of life, including pain, fasciculations, sialorrhea, pseudobulbar affect, spasticity, cramps, depression, anxiety, insomnia and fatigue. Several clinical trials have explored treatment options for sialorrhea ${ }^{42}$ and pseudobulbar affect. ${ }^{43}$ However, management of most ALS symptoms has not been rigorously evaluated. As a consequence, most of the recommendations for symptom management were decided by expert consensus and supported by treatment suggestions made in the ALS and palliative care literature. Cost and access to treatments affected our ordering of the recommendations and were weighted more highly than direct evidence if an evidencesupported treatment was expensive. Our recommendations did not include the option of cannabis to treat specific ALS symptoms, because of lack of evidence in the literature. However, the working group is aware that cannabis is being used to manage several ALS symptoms.

\section{Dysarthria}

The ability to communicate thoughts and needs to others is vitally important to individuals. ALS often impairs the ability to communicate verbally because of dysarthria. ${ }^{2}$ Multiple available interventions can be initiated to support communication, including low-tech options, such as letter- or picture-boards, and hightech options, such as speech synthesizers and eye-gaze tracking. As individuals with ALS experience loss of function, some modes of communication may no longer be viable. Providing access to different modes of communication, including social media, can allow independence, participation and better quality of life. ${ }^{44}$ Communication devices may also benefit caregivers, as the burden on caregivers was found to be reduced when patients used an eye-tracking assistive device. ${ }^{45}$

\section{Exercise}

Research on exercise in ALS has not demonstrated harm and some evidence has suggested that there is a potential benefit for patients in terms of function and quality of life. ${ }^{46,47} \mathrm{~A}$ personalized exercise program, including strength and aerobic training, should be encouraged for patients who are able to participate. A regular stretching and range-of-motion program is recommended for management of spasticity and pain, and prevention of contractures.

\section{Cognition and behaviour}

Detectable frontotemporal dysfunction can occur in about $50 \%$ of patients with ALS. ${ }^{3}$ The frontotemporal dysfunction can present with cognitive impairment or behavioural impairment, which in $20 \%$ of patients is severe enough to reach criteria for dementia. ${ }^{3}$ 
Although there are many tools available to screen for cognitive or behavioural impairment, there is no standard tool to use. At this time, there are no effective drug treatments for cognitive or behavioural impairment in ALS. A multidisciplinary approach can be considered to manage particularly problematic behaviours.

The presence of executive dysfunction or dementia in ALS is associated with poor survival. ${ }^{48}$ The presence of cognitive or behavioural impairment should not necessarily preclude implementing the recommendations for noninvasive ventilation or gastrostomy insertion. However, the challenges of intervention compliance with cognitive or behavioural impairment should be discussed with the patient and family before deciding to proceed with an intervention.

\section{Caregivers}

Informal caregivers are affected by caring for the person with ALS. Many studies have demonstrated the impact of ALS on caregiver quality of life and the correlates of caregiver burden, ${ }^{49}$ but also the value of caregiving. ${ }^{50}$ Advanced disability (a low ALSFRS-R score) and cognitive impairment increase caregiver strain. ${ }^{51}$ Interventions to mitigate the impact on caregivers have been insufficiently studied to make specific recommendations. People with ALS are aware of and affected by the burden on their caregivers. ${ }^{52}$ Health care providers, therefore, need to be attentive to the physical and emotional well-being of the caregivers, and involve them in planning for the impact of ALS on both the patient and themselves.

\section{Palliative care}

Expert opinion supports early integration of palliative care for patients with ALS. ${ }^{8,53}$ However, palliative and end-of-life care are sensitive topics and variably received by patients. ${ }^{54}$ Therefore, early introduction of palliative care must be initiated with consideration of the patient's evolving needs and expectations..$^{55} \mathrm{At}$ the very least, experts have advocated that it is appropriate to initiate discussions about palliative care if the topic is raised by patients or caregivers, and if there are indications of advanced disease or disability. ${ }^{53}$

Advance care planning helps establish care preferences before the disease is advanced and communication is impaired. There is evidence to suggest that these discussions are best initiated when the patient has accepted that death will eventually occur. ${ }^{56}$ However, there is a general reluctance among clinicians to broach the topic, as it may be perceived to indicate the imminence of death. ${ }^{57}$ Standardized tools for advance care planning are thought to be useful for stimulating these discussions, rather than for generating specifics of an advanced directive. ${ }^{56,58,59}$ Thus, discussions may be integrated into routine ALS follow-up to invite open conversation, and should take into account the patient's readiness and style of decision-making.

Medical assistance in dying (MAiD) was legalized in Canada in 2016. We have made specific recommendations as to how requests for MAiD should be addressed, both to support patient choice at end of life, and to provide guidance in this new practice, which may be a source of clinical uncertainty and discomfort to some practitioners.
We also present recommendations on the potential option of organ donation at the time of death and the process that should be followed for donation.

\section{Methods}

The concept for this guideline was concurrently fostered by ALS Canada and Canadian ALS clinicians within the Canadian ALS Research Network (CALS; now merged with ALS Canada). The guideline was developed using the Guideline International Network-McMaster Guideline Development Checklist, ${ }^{60}$ for guidance on all aspects of guideline development, including planning, formulation of recommendations, implementation and evaluation. A complete description of the guideline methodology is available in the full guideline (www.als.ca/bpr-appendix).

\section{Guideline panel composition}

A working group of 13 Canadian ALS clinicians (the authors), chaired by C.S., led the development of this guideline. Neurologists and physiatrists who were active in the Canadian ALS Research Network and could represent the geographic diversity of Canada were invited to participate in the working group. Clinicians with previous experience with guideline development were particularly encouraged to participate. The working group also included a gastroenterologist (D.L.) and a respirologist (A.T.) with ALS expertise. Early in the guideline development process, 2 other Canadian ALS clinicians were involved, but they removed themselves from the project because of the time commitments required.

\section{Selection of key questions}

In 2014, we selected clinical questions of interest for the guideline by surveying clinicians and staff at all 19 Canadian multidisciplinary ALS clinics via an emailed survey. The survey included a list of the key questions used to develop the American Academy of Neurology (AAN) Practice Parameters ${ }^{6,7}$ and European Network for the Cure of ALS guideline, ${ }^{8}$ as well as additional questions that members of the working group had derived based on their own clinical experience. We asked survey participants to rate the importance of these questions for inclusion in the guideline.

Questions included in the literature review were those questions rated highly by participants on the survey; the working group further refined these questions. The selected clinical questions were grouped by topic including communication of diagnosis, disease-modifying therapy, multidisciplinary care, respiratory management, nutrition management, symptom management, cognitive impairment, risk of venous thromboembolism (VTE), exercise, palliative care and caregiver support.

\section{Literature search}

In 2015, the Centre for Effective Practice, a consulting firm with substantial guideline development experience, conducted literature searches for the selected clinical questions using MEDLINE, Embase and CINAHL databases. The centre developed the search terms for each clinical question through review of the search terms 
that had been used for the AAN guideline ${ }^{6,7}$ and in consultation with the working group. The centre performed a second literature search in December 2018 to search for papers published after the initial search in 2015. For clinical questions addressed in the AAN guideline, literature searches were restricted to publications dated from 2007 to December 2018. For new clinical questions that had not been addressed in the AAN guideline or European Network for the Cure of ALS guideline, literature searches were restricted to publications dated from 1998 to December 2018. The search strategies are available in Appendix A of the full guideline (www.als.ca/bpr-appendix).

\section{Quality assessment}

The working group was divided into topic groups, with 2 members per group. For questions grouped under a major topic, 2 members of each topic group screened the retrieved abstracts separately based on the inclusion criteria and relevance to the clinical question. Inclusion criteria included published ALS guidelines, ventilation guidelines, RCTs, case-control studies, cohort studies, systematic reviews and meta-analyses. Publications had to be published in English or French and available in full text. Single-case reports, review articles, publications available only in abstract or proceeding forums, and thesis data not published elsewhere were excluded. Publications felt by at least 1 of the abstract reviewers to meet the inclusion criteria were reviewed in full by the topic group for inclusion criteria and data quality and assigned a class of evidence based on criteria modified from the AAN Clinical Practice Guideline Process Manual (2011 Edition) to rate therapeutic studies (Box 1 ). The evidence tables are available in Appendix B of the full guideline (www.als.ca/ bpr-appendix).

\section{Development of recommendations}

The working group met regularly at face-to-face meetings at least annually in Toronto, and through regular group teleconferences to discuss the specifics of guideline statements. Each topic group drafted preliminary guideline statements for each clinical question after considering previously published guideline statements $^{6-8}$ and updated evidence.

The working group reviewed these draft statements and refined them on an iterative basis, ideally until consensus was obtained. If consensus could not be reached among the working group, we agreed that a decision would be made based on a twothirds majority (66\%) vote. However, there was consensus on all statements and so no vote was held.

We assigned each statement a level of evidence, which included the option of expert consensus (Box 2). The working group felt strongly that in the absence of published evidence, best practice recommendations based on expert consensus should be included, rather than no recommendation provided. Given that there is limited evidence from clinical trials to direct care in ALS, the working group members thought it important that the recommendations be a practical guide to the care of patients with ALS, rather than simply a review of the evidence. We made expert consensus statements based on nonclinical trial literature in ALS, evidence in other diseases or current Canadian ALS clinical practice. We discussed the order of the statements in the recommendations table at length to reflect their clinical

Box 1: Criteria for rating therapeutic studies ${ }^{\star 61}$

\section{Class}

\section{Description}

- Randomized controlled clinical trial (RCT) in a representative population

- Masked or objective outcome assessment

- Relevant baseline characteristics are presented and substantially equivalent between treatment groups, or there is appropriate statistical adjustment for differences

- Also required:

a. Concealed allocation

b. Primary outcome(s) clearly defined

c. Exclusion and inclusion criteria clearly defined

d. Adequate accounting for dropouts (with at least $80 \%$ of enrolled participants completing the study) and crossovers with numbers sufficiently low to have minimal potential for bias

II - Cohort study meeting criteria a-d (see class I) or an RCT that lacks 1 or 2 criteria b-d (see class I)

- All relevant baseline characteristics are presented and substantially equivalent among treatment groups, or there is appropriate statistical adjustment for differences

- Masked or objective outcome assessment

III - Controlled studies (including well-defined natural history controls or patients serving as their own controls)

- A description of major confounding differences between treatment groups that could affect outcome

- Outcome assessment masked, objective or performed by someone who is not a member of the treatment team

IV Did not include patients with the disease

- Did not include patients receiving different interventions

- Undefined or unaccepted interventions or outcome measures

- No measures of effectiveness or statistical precision presented or calculable

*Modified with permission from AAN (American Academy of Neurology). 2011. Clinical practice guideline process manual, 2011 Ed. St. Paul (MN): The American Academy of Neurology; 2011. Available online at www.aan.com/siteassets/home-page/policy-and-guidelines/guidelines/about-guidelines/11guidelinedevmanual_v408_web.pdf. Accessed 2020 Sept. 11. 
importance and the order in which a practitioner would consider interventions when caring for patients.

After the statements we developed for the respiratory questions had obtained consensus support from our working group, the Canadian Thoracic Society (CTS) published a guideline on the respiratory care of patients with ALS. ${ }^{35}$ The working group decided it was important for our recommendations to be consistent with this guideline. To accomplish this, we compared each of the recommendations in the CTS guideline with our draft statements. Our working group accepted most of the CTS recommendations without changes, apart from slight wording alterations for consistency. We asked the CTS ALS committee to review our suggested statements, including those expert consensus statements where questions deemed important in our survey had not been addressed by the CTS guideline; their feedback led to some minor changes in our wording.

\section{Review process}

We developed an executive summary of the guideline statements and the working group reviewed it. When the working group was satisfied with the recommendation statements, including the wording, order and evidence ranking, we emailed this executive draft summary to members of the Canadian ALS Research Network (which includes all multidisciplinary ALS clinics in Canada) and topic experts external to the working group (i.e., with expertise in gastroenterology, respirology, palliative care and physiatry) for open-ended feedback. We asked the ALS clinics to share the executive draft summary with their allied health staff and request additional open-ended feedback from them as well. The working group discussed each comment received to determine whether changes were required to the recommendation statements and if so, how the statements should be revised.

Using the revised executive draft summary, a second round of external review followed, in which we asked key stakeholders within each provincial ALS society to participate. We emailed the revised executive draft summary to each of the provincial ALS societies along with an attached survey with open-ended questions. We asked each society to solicit feedback from its members, including 1 patient living with ALS in its province. All comments received were individually considered by the working group and changes were implemented at its discretion through a robust discussion about the feedback. The changes made according to the feedback received involved wording changes for the most part. We made no substantial changes.
We prepared a complete version of the guideline and all working group members reviewed it for final approval.

\section{Management of competing interests}

All members of the working group performed their tasks voluntarily and did not receive honoraria for their involvement. ALS Canada and the Canadian ALS Research Network funded the development of the guideline, including travel for face-to-face meetings and preparation of the manuscript for publication.

ALS Canada is a grassroots donor-funded organization and part of the funding for this project came from donations during the Ice Bucket Challenge. ALS Canada assisted with logistic support but did not contribute to the content of the recommendations. The Canadian ALS Research Network was a nonprofit organization of ALS clinicians and researchers formed to increase clinical ALS research in Canada and funded by stipends given by biotechnology companies to review clinical trial proposals for Canadian ALS clinics (it has subsequently merged with ALS Canada). Although members of CALS participated in the development of the guideline, CALS had no role in approving guideline recommendations.

We discussed competing interest management during the planning phase of the guideline; competing interests were defined as a financial relationship with a company. At that time, there was only 1 drug approved by Health Canada for the treatment of ALS: riluzole. None of the working group members had conflicts related to the drug riluzole, which has been available for more than 20 years. We solicited other potential conflicts of interest from the working group at the beginning of this project, and no conflicts were present.

In 2017, during the guideline development process, the US Food and Drug Administration approved edaravone to treat ALS. Its manufacturer, Mitsubishi Tanabe Pharma, sponsored scientific advisory committees regarding the use of edaravone in patients with ALS in Canada. Health Canada approved the drug in October 2018 and it became commercially available in Canada in November 2019. Some working group members sat on the Mitsubishi Tanabe Pharma scientific advisory committees for edaravone (C.S., M.C., A.I., W.J., C.O., K.S., L Z.), which they disclosed to the working group. All members of the working group discussed at length the statements in this guideline regarding edaravone. During review of the draft guideline, feedback from members of the Canadian ALS Research Network and key stakeholders regarding the edaravone statements was deliberated by working group members who did not have conflicts of interest with

Box 2: Criteria for levels of evidence in guideline recommendations*

\section{Level}

A

B

C

Expert consensus

\section{Type of evidence}

At least 2 consistent class I studies

At least 1 class I study or 2 consistent class II studies

At least 1 class II study or 2 consistent class III studies

Consensus among Canadian amyotrophic lateral sclerosis clinical experts where evidence meeting criteria for Level A through Level C is lacking

*See Box 1 for definitions of study classes. 
Mitsubishi Tanabe Pharma, defined as having received any honoraria from the company. Final decisions regarding the edaravone statements were made by working group members without potential conflicts. Other than edaravone, there are no other potential conflicts with the statements in this guideline.

\section{Implementation}

These best practice recommendations are a resource to guide the care of patients with ALS across Canada. The guideline will be made publicly accessible through the ALS Canada website (www.als.ca). ALS Canada will also support the dissemination of the guideline among members of the ALS community, including clinicians, allied health professionals, researchers, patients and their caregivers, through distribution to provincial ALS societies, the Canadian ALS Research Network and attendees of the annual ALS Canada Research Forum. Directors of ALS clinics and ALS clinicians will be encouraged to present the guideline to their clinic teams and relevant stakeholders within their communities. ALS Canada will assist the guideline authors with producing 1-page summary documents of some key clinical areas of the guideline for dissemination to stakeholders.

The working group would support a health impact project assessing patient survival, patient-perceived quality of life and other specific outcomes after the implementation of the guideline compared with before its publication.

The working group expects that evidence to support ALS management will evolve over time and anticipates that the recommendations will have to be revised approximately every 5 years.

\section{Other guidelines}

Several ALS clinical practice guidelines have been published in countries other than Canada, including the AAN Practice Parameters (2009), ${ }^{6,7}$ the European Federation of Neurological Societies guideline on the clinical management of amyotrophic lateral sclerosis (2012), ${ }^{8}$ and the motor neurone disease assessment and management guideline developed by England's National Institute for Health and Care Excellence (2016). ${ }^{9}$

One of the goals for the Canadian guideline was to update the existing North American guidelines, specifically the 2009 AAN recommendations. ${ }^{6,7}$ As described in the Methods section, literature searches for this Canadian guideline on clinical questions addressed in the 2009 AAN recommendations were restricted to new evidence only (i.e., after 2007), and all evidence was classified using AAN criteria.

In the AAN guideline, recommendations had to be supported by evidence; thus, no guidance was provided in the absence of evidence (e.g., using expert consensus). In contrast, the European Federation of Neurological Societies guideline provided consensus recommendations in the absence of evidence. We also resolved to offer guidance based on expert consensus in the absence of evidence.

Another goal for the Canadian guideline was to address ALS issues not covered in other guidelines. The European Federation of Neurological Societies guideline did not address several issues for patients with ALS that are important in Canada, such as medication alignment and MAiD. Similarly, guidance on some ALS issues, such as disease-modifying treatments and exercise, was not provided in the National Institute for Health and Care Excellence guideline.

As discussed earlier, the CTS published a guideline on home mechanical ventilation for patients with ALS in early 2019. ${ }^{35}$ In collaboration with the CTS, we ensured that our recommendations for respiratory management were consistent with recommendations in the CTS guideline, but added some consensus recommendations (e.g., on airway clearance).

\section{Gaps in knowledge}

This guideline confirms that high-quality evidence is lacking for most topics in ALS management; most recommendations provided are based on expert consensus among the working group. The need for further research in ALS management remains, and more evidence-based recommendations will be critical for improving the standards of patient care in Canada and internationally. This guideline can help point the clinical research community, nationally and internationally, to areas of research priorities on disease management.

We acknowledge that we were not able to cover all topics of ALS management in this guideline and that subsequent revisions could include topics not currently covered.

\section{Conclusion}

We hope that the development of the first Canadian ALS guideline is an important step forward for improving the lives of patients with ALS living in Canada. The predominance of expert consensus statements relative to evidence-based statements in this guideline not only highlights the need for more research in ALS management but also emphasizes the challenges ALS clinicians face in managing patients with a severe disabling disease. This guideline will enable ALS clinics across Canada to meet a common national standard, and to adapt as this standard continues to evolve over time. In doing so, ALS clinicians can offer the best possible care to their patients and help them to navigate this exceedingly complex and devastating disease.

\section{References}

1. Brown RH, Al-Chalabi A. Amyotrophic lateral sclerosis. N Engl J Med 2017;377:162-72.

2. van Es MA, Hardiman O, Chio A, et al. Amyotrophic lateral sclerosis. Lancet 2017;390:2084-98.

3. Strong MJ, Abrahams S, Goldstein LH, et al. Amyotrophic lateral sclerosis frontotemporal spectrum disorder (ALS-FTSD): Revised diagnostic criteria. Amyotroph Lateral Scler Frontotemporal Degener 2017;18:153-74.

4. Shoesmith C. A recipe for ALS. Can J Neurol Sci 2008;35:125-126.

5. Health Canada approves new drug to treat patients with amyotrophic lateral sclerosis (ALS) [press release]. Ottawa: Health Canada; 2018 Oct. 4.

6. Miller RG, Jackson CE, Kasarskis EJ, et al. Practice parameter update: the care of the patient with amyotrophic lateral sclerosis: drug, nutritional, and respiratory therapies (an evidence-based review): report of the Quality Standards Subcommittee of the American Academy of Neurology. Neurology 2009;73:1218-26. 
7. Miller RG, Jackson CE, Kasarskis EJ, et al. Practice parameter update: the care of the patient with amyotrophic lateral sclerosis: multidisciplinary care, symptom management, and cognitive/behavioral impairment (an evidence-based review): report of the Quality Standards Subcommittee of the American Academy of Neurology. Neurology 2009;73:1227-33.

8. The EFNS Task Force on Diagnosis and Management of Amyotrophic Lateral Sclerosis, Andersen PM, Abrahams S, Borasio GD, et al. EFNS guidelines on the Clinical Management of Amyotrophic Lateral Sclerosis (MALS) - revised report of an EFNS task force. Eur J Neurol 2012;19:360-75.

9. Motor neurone disease: assessment and management (NG42). London (UK): National Institute for Healthcare and Excellence (NICE); 2016.

10. Peters M, Fitzpatrick R, Doll H, et al. Patients' experiences of health and social care in long-term neurological conditions in England: a cross-sectional survey. J Health Serv Res Policy 2013;18:28-33.

11. Abdulla S, Vielhaber S, Machts J, et al. Information needs and informationseeking preferences of ALS patients and their carers. Amyotroph Lateral Scler Frontotemporal Degener 2014;15:505-12.

12. Ang K, Umapathi T, Tong J, et al. Healthcare needs of patients with amyotrophic lateral sclerosis (ALS) in Singapore: a patient-centred qualitative study from multiple perspectives. J Palliat Care 2015;31:150-7.

13. Chiò A, Montuschi A, Cammarosano S, et al. ALS patients and caregivers communication preferences and information seeking behaviour. Eur J Neurol 2008;15:55-60.

14. Miller RG, Mitchell JD, Moore DH. Riluzole for amyotrophic lateral sclerosis (ALS)/motor neuron disease (MND). Cochrane Database Syst Rev 2012; (3):CD001447.

15. Rooney J, Byrne S, Heverin M, et al. Survival analysis of irish amyotrophic lateral sclerosis patients diagnosed from 1995-2010. PLoS One 2013;8:e74733.

16. Stevic Z, Kostic-Dedic S, Peric S, et al. Prognostic factors and survival of ALS patients from Belgrade, Serbia. Amyotroph Lateral Scler Frontotemporal Degener 2016;17:508-14.

17. Georgoulopoulou E, Fini N, Vinceti M, et al. The impact of clinical factors, riluzole and therapeutic interventions on ALS survival: a population-based study in Modena, Italy. Amyotroph Lateral Scler Frontotemporal Degener 2013; 14:338-45.

18. Wei Q, Chen X, Zheng Z, et al. The predictors of survival in Chinese amyotrophic lateral sclerosis patients. Amyotroph Lateral Scler Frontotemporal Degener 2015;16:237-44.

19. Chen L, Liu X, Tang L, et al. Long-term use of riluzole could improve the prognosis of sporadic amyotrophic lateral sclerosis patients: a real-world cohort study in China. Front Aging Neurosci 2016;8:246.

20. Mandrioli J, Malerba SA, Beghi E, et al. Riluzole and other prognostic factors in ALS: a population-based registry study in Italy. J Neurol 2018;265:817-27.

21. Sívori M, Rodriguez GE, Pascansky D, et al. Outcome of sporadic amyotrophic lateral sclerosis treated with non-invasive ventilation and riluzole. Medicina ( $B$ Aires) 2007;67:326-30

22. Lee CT, Chiu YW, Wang KC, et al. Riluzole and prognostic factors in amyotrophic lateral sclerosis long-term and short-term survival: a population-based study of 1149 cases in Taiwan. J Epidemiol 2013;23:35-40.

23. Watanabe H, Atsuta N, Nakamura R, et al. Factors affecting longitudinal functional decline and survival in amyotrophic lateral sclerosis patients. Amyotroph Lateral Scler Frontotemporal Degener 2015;16:230-6.

24. Calvo A, Moglia C, Lunetta C, et al. Factors predicting survival in ALS: a multicenter Italian study. J Neurol 2017;264:54-63.

25. Keren N, Scott KM, Tsuda M, et al. Evidence of an environmental effect on survival in ALS. Amyotroph Lateral Scler Frontotemporal Degener 2014;15:528-33.

26. Fang T, Al Khleifat A, Meurgey JH, et al. Stage at which riluzole treatment prolongs survival in patients with amyotrophic lateral sclerosis: a retrospective analysis of data from a dose-ranging study. Lancet Neurol 2018;17:416-22.

27. Chen X, Wei QQ, Chen Y, et al. Clinical staging of amyotrophic lateral sclerosis in Chinese patients. Front Neurol 2018;9:442.

28. Zoccolella S, Beghi E, Palagano G, et al. Riluzole and amyotrophic lateral sclerosis survival: a population-based study in southern Italy. Eur J Neurol 2007;14:262-8.

29. Abe K, Itoyama Y, Sobue G, et al. Confirmatory double-blind, parallel-group, placebo-controlled study of efficacy and safety of edaravone ( $\mathrm{MCl}-186)$ in amyotrophic lateral sclerosis patients. Amyotroph Lateral Scler Frontotemporal Degener. 2014;15:610-7.
30. Writing Group, Edaravone ALSSG. Safety and efficacy of edaravone in well defined patients with amyotrophic lateral sclerosis: a randomised, doubleblind, placebo-controlled trial. Lancet Neurol. 2017;16:505-12.

31. Rooney J, Byrne S, Heverin M, et al. A multidisciplinary clinic approach improves survival in ALS: a comparative study of ALS in Ireland and Northern Ireland. J Neurol Neurosurg Psychiatry 2015;86:496-501.

32. Traynor BJ, Alexander M, Corr B, et al. Effect of a multidisciplinary amyotrophic lateral sclerosis (ALS) clinic on ALS survival: a population-based study, 1996-2000. J Neurol Neurosurg Psychiatry 2003;74:1258-61.

33. Van den Berg JP, Kalmijn S, Lindeman E, et al. Multidisciplinary ALS care improves quality of life in patients with ALS. Neurology 2005;65:1264-7.

34. Geronimo A, Wright C, Morris A, et al. Incorporation of telehealth into a multidisciplinary ALS Clinic: feasibility and acceptability. Amyotroph Lateral Scler Frontotemporal Degener 2017;18:555-61.

35. Rimmer KP, Kaminska M, Nonoyama M, et al. Home mechanical ventilation for patients with amyotrophic lateral sclerosis: a Canadian Thoracic Society clinical practice guideline. Can J Respir Cri Care Sleep Med 2019;3:9-27.

36. Gonzalez Calzada N, Prats Soro E, Mateu Gomez L, et al. Factors predicting survival in amyotrophic lateral sclerosis patients on non-invasive ventilation. Amyotroph Lateral Scler Frontotemporal Degener 2016;17:337-42.

37. Silva LB, Mourao LF, Silva AA, et al. Effect of nutritional supplementation with milk whey proteins in amyotrophic lateral sclerosis patients. Arq Neuropsiquiatr 2010;68:263-8.

38. Dorst J, Cypionka J, Ludolph AC. High-caloric food supplements in the treatment of amyotrophic lateral sclerosis: a prospective interventional study. Amyotroph Lateral Scler Frontotemporal Degener 2013;14:533-6.

39. Wills AM, Hubbard J, Macklin EA, et al. Hypercaloric enteral nutrition in patients with amyotrophic lateral sclerosis: a randomised, double-blind, placebocontrolled phase 2 trial. Lancet 2014;383:2065-72.

40. Gladman M, Dehaan M, Pinto H, et al. Venous thromboembolism in amyotrophic lateral sclerosis: a prospective study. Neurology 2014;82:1674-7.

41. Qureshi MM, Cudkowicz ME, Zhang H, et al. Increased incidence of deep venous thrombosis in ALS. Neurology 2007;68:76-7.

42. Jackson CE, Gronseth G, Rosenfeld J, et al. Randomized double-blind study of botulinum toxin type B for sialorrhea in ALS patients. Muscle Nerve 2009; 39:137-43.

43. Brooks BR, Thisted RA, Appel SH, et al. Treatment of pseudobulbar affect in ALS with dextromethorphan/quinidine: a randomized trial. Neurology 2004; 63:1364-70.

44. Caron J, Light J. "My world has expanded even though i'm stuck at home": experiences of individuals with amyotrophic lateral sclerosis who use augmentative and alternative communication and social media. Am J Speech Lang Pathol 2015;24:680-95.

45. Hwang $\mathrm{C}-\mathrm{S}$, Weng $\mathrm{H}-\mathrm{H}$, Wang L-F, et al. An eye-tracking assistive device improves the quality of life for ALS patients and reduces the caregivers' burden. J Mot Behav 2014;46:233-8.

46. Clawson LL, Cudkowicz M, Krivickas L, et al. A randomized controlled trial of resistance and endurance exercise in amyotrophic lateral sclerosis. Amyotroph Lateral Scler Frontotemporal Degener 2018;19:250-8.

47. Lunetta C, Lizio A, Sansone VA, et al. Strictly monitored exercise programs reduce motor deterioration in ALS: preliminary results of a randomized controlled trial. J Neurol 2016;263:52-60.

48. Elamin M, Phukan J, Bede P, et al. Executive dysfunction is a negative prognostic indicator in patients with ALS without dementia. Neurology 2011; 76:1263-9.

49. Burke T, Hardiman O, Pinto-Grau M, et al. Longitudinal predictors of caregiver burden in amyotrophic lateral sclerosis: a population-based cohort of patientcaregiver dyads. J Neurol 2018;265:793-808.

50. Lerum SV, Solbraekke KN, Frich JC. Family caregivers' accounts of caring for a family member with motor neurone disease in Norway: a qualitative study. BMC Palliat Care 2016;15:22.

51. Watermeyer TJ, Brown RG, Sidle KC, et al. Impact of disease, cognitive and behavioural factors on caregiver outcome in amyotrophic lateral sclerosis. Amyotroph Lateral Scler Frontotemporal Degener 2015;16:316-23.

52. Foley G, Timonen V, Hardiman O. "I hate being a burden": the patient perspective on carer burden in amyotrophic lateral sclerosis. Amyotroph Lateral Scler Frontotemporal Degener 2016;17:351-7. 
53. Mitsumoto H, Bromberg M, Johnston W, et al. Promoting excellence in end-of-life care in ALS. Amyotroph Lateral Scler Other Motor Neuron Disord 2005;6:145-54.

54. Ray RA, Brown J, Street AF. Dying with motor neurone disease, what can we learn from family caregivers? Health Expect 2014;17:466-76.

55. Lulé D, Nonnenmacher S, Sorg S, et al. Live and let die: existential decision processes in a fatal disease. $J$ Neurol 2014;261:518-25.

56. Murray L, Butow PN, White K, et al. Advance care planning in motor neuron disease: A qualitative study of caregiver perspectives. Palliat Med 2016;30:471-8.

57. Burchardi N, Rauprich $\mathrm{O}$, Hecht $\mathrm{M}$, et al. Discussing living wills. A qualitative study of a German sample of neurologists and ALS patients. J Neurol Sci 2005;237:67-74.
58. Preston H, Fineberg IC, Callagher P, et al. The preferred priorities for care document in motor neurone disease: views of bereaved relatives and carers. Palliat Med 2012;26:132-8

59. Sulmasy DP, Terry PB, Weisman CS, et al. The accuracy of substituted judgments in patients with terminal diagnoses. Ann Intern Med 1998;128:621-9.

60. Schünemann HJ, Wiercioch W, Etxeandia I, et al. Guidelines 2.0: systematic development of a comprehensive checklist for a successful guideline enterprise. CMAJ 2014;186:E123-42.

61. Clinical practice guideline process manual. St. Paul (MN): AAN (American Academy of Neurology); 2011.
Competing interests: Christen Shoesmith, Aaron Izenberg, Wendy Johnston, Colleen O'Connell, Kerri Schellenberg and Lorne Zinman were members of a scientific advisory committee for Radicava (edaravone; Mitsubishi Tanabe Pharma Canada). Christen Shoesmith reports being a site principal investigator for several multicentre amyotrophic lateral sclerosis (ALS) clinical trials. In the last 36 months, Dr. Shoesmith has participated in clinical trials sponsored by Biogen, Cytokinetics, ALS Pharma and Orphazyme. Marvin Chum reports receiving a grant from Bernice Ramsay ALS Clinical Research Fellowship, outside the submitted work. Aaron Izenberg reports receiving personal fees from Biogen, Roche, Alnylam, Genzyme, Takeda and Mitsubishi Tanabe Pharma, outside the submitted work. No other competing interests were declared.

This article has been peer reviewed.

Affiliations: London Health Sciences Centre (Shoesmith), London, Ont.; Sunnybrook Health Sciences Centre (Abrahao, Izenberg, Tandon, Zinman), Toronto, Ont.; Dalhousie University (Benstead, Leddin), Halifax, NS; McMaster University (Chum), Hamilton, Ont.; CHU de Québec-Université Laval (Dupre), Québec, Que.; University of Alberta (Johnston, Kalra), Edmonton, Alta.; Stan Cassidy Centre for Rehabilitation (O'Connell), Fredericton, NB; University of Saskatchewan (Schellenberg), Saskatoon, Sask.
Contributors: All of the authors contributed to the conception and design of the work, and the acquisition, analysis and interpretation of data. All of the authors drafted the manuscript, revised it critically for important intellectual content, gave final approval of the version to be published and agreed to be accountable for all aspects of the work.

Funding: Funding for the development of the recommendations was provided by the ALS Society of Canada and the Canadian ALS Research Network.

Acknowledgements: The writing group thanks ALS Canada for its ongoing support for the development of these guidelines. The authors also acknowledge Ms. Vanessa Blount, who helped with the initial coordination of the guidelines, Dr. David Taylor and Ms. Colleen Doyle for logistical support, and Ms. Trisha Rao, who helped with the editing of the document. The Canadian ALS Research Network and Federation partners also provided critical feedback with respect to the wording of the guidelines and manuscript. Dr. Ikhlass Salem Haj, Dr. Lawrence Korngut and Dr. Hannah Briemberg also provided substantial assistance in the development of these recommendations.

Correspondence to: Christen Shoesmith, Christen.Shoesmith@lhsc.on.ca 